\title{
Top k 2-Clubs in a Network: A Genetic Algorithm
}

\author{
Mauro Castelli (1), Riccardo Dondi (2), Sara Manzoni (3), \\ Giancarlo Mauri (3), and Italo Zoppis (3) \\ 1 NOVA Information Management School (NOVA IMS), Universidade Nova de Lisboa, \\ Campus de Campolide, 1070-312 Lisboa, Portugal \\ 2 Universit 'a degli Studi di Bergamo, Bergamo, Italy \\ 3 Universit 'a degli Studi di Milano-Bicocca, Milano - Italy \\ mcastelli@novaims.unl.pt, riccardo.dondi@unibg.it,sara.manzoni@unimib.it, \\ giancarlo.mauri@unimib.it,italo.zoppis@unimib.it
}

This is the Author Peer Reviewed version of the following conference paper published by Springer:

Castelli, M., Dondi, R., Manzoni, S., Mauri, G., \& Zoppis, I. (2019). Top k 2clubs in a network: A genetic algorithm. In J. J. Dongarra, J. M. F. Rodrigues, P. J. S. Cardoso, J. Monteiro, R. Lam, V. V. Krzhizhanovskaya, M. H. Lees, ... P. M. A. Sloot (Eds.), Computational Science. ICCS 2019: 19th International Conference, 2019, Proceedings (Vol. 5, pp. 656-663). (Lecture Notes in Computer Science (including subseries Lecture Notes in Artificial Intelligence and Lecture Notes in Bioinformatics); Vol. 11540 LNCS). Springer Verlag. https://doi.org/10.1007/978-3-030-22750-0_63, which has been published in final form at https://doi.org/10.1007/978-3-030-22750-0_63

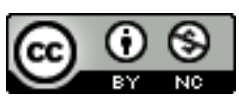

This work is licensed under a Creative Commons Attribution-NonCommercial 4.0 International License. 


\title{
Top $k$ 2-Clubs in a Network: A Genetic Algorithm
}

\author{
Mauro Castelli ${ }^{1}$, Riccardo Dondi ${ }^{2}$, Sara Manzoni $^{3}$, \\ Giancarlo Mauri ${ }^{3}$, and Italo Zoppis ${ }^{3}$ \\ 1 NOVA Information Management School (NOVA IMS), Universidade Nova de Lisboa, \\ Campus de Campolide, 1070-312 Lisboa, Portugal \\ 2 Università degli Studi di Bergamo, Bergamo, Italy \\ 3 Università degli Studi di Milano-Bicocca, Milano - Italy \\ mcastelli@novaims.unl.pt, riccardo.dondidunibg.it, sara.manzonidunimib.it, \\ giancarlo.mauri@unimib.it, italo.zoppis@unimib.it
}

\begin{abstract}
The identification of cohesive communities (dense subgraphs) is a typical task applied to the analysis of social and biological networks. Different definitions of communities have been adopted for particular occurrences. One of these, the 2-club (dense subgraphs with diameter value at most of length 2) has been revealed of interest for applications and theoretical studies. Unfortunately, the identification of 2-clubs is a computationally intractable problem, and the search of approximate solutions (at a reasonable time) is therefore fundamental in many practical areas. In this article, we present a genetic algorithm based heuristic to compute a collection of Top $k$ 2-clubs, i.e., a set composed by the largest $k$ 2-clubs which cover an input graph. In particular, we discuss some preliminary results for synthetic data obtained by sampling Erdös-Rényi random graphs.
\end{abstract}

Keywords: Community optimization · 2-club maximization · Genetic Algorithms - Graphs.

\section{Introduction}

The identification of communities within a network is a typical task that has been widely applied in different contexts. In particular, dense subgraphs (i.e., cohesive communities) have perceived the attention of the scientific literature oriented to the analysis of social $[18,1,16,19]$ and biological networks [20,3]. A standard approach to compute dense subgraphs is focused on the identification of structures known as cliques: complete subgraphs whose vertices are pairwise connected by edges. However, the use of a clique is too binding for specific applications. For example, a critical issue arises when missing data are persistent in the considered analysis. In this case, the missing information does not allow to represent all links of a dense community, thus requiring to search alternative notions of dense subgraphs. For this reason, different definitions of community have been introduced in literature "by relaxing" to some extent the concept of clique (see, e.g., [14] for the concept of relaxed clique).

In this paper, we focus on a distance-based relaxation of the clique model. In other words, instead of seeking structures where distances between pairs of vertices are equal to 1 (i.e., cliques), we will consider dense subgraphs where the distance between vertices can be at most s. Such a structure is generally known as s-club. In particular, due to

ICCS Camera Ready Version 2019

To cite this paper please use the final published version:

DOI $10.1007 / 978-3-030-22750-0 \_63$ 


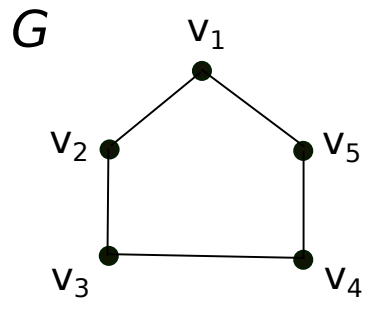

Fig. 1. The figure shows a 2 -club of 5 vertices. Notice that, each subgraph of 4 vertices is not a 2-club.

the importance of this problem for social [18, 1, 16, 19] and biological networks [20,3], we will consider the case of $s=2$.

From the computational point of view, the literature concerning $s$-clubs has mainly focused on the identification of $s$-clubs of maximum size (a problem known as Max-s$(l u b)$, and its complexity has been extensively studied. Although the results have shown the NP-hardness of the problem for each $s \geq 1$ [6], polynomial-time approximation algorithms, with factor $|V|^{1 / 2}$, are available for every $s \geq 2$ [2]. Recently, the problem has been investigated for restricted graph classes [13, 12], and even for its parameterized complexity. The problem has been shown to be fixed-parameter tractable, when the parameter is the size of the sought $s$-club [21, 15,7].

In many real applications, the objective is to find a set of cohesive subgraphs of the original input graph (rather than a single subgraph covering the input). Following the approach proposed in [11], this paper considers the problem of computing the set of largest $k 2$-clubs, with $k \geq 1$. We will denote this problem as Top $k$-2-clubs. Notice that other problems that seek for 2-clubs have been considered recently. In [10], it is considered the problem of finding a maximum set of disjoint $s$-clubs of at least a given size, while in [8] it is considered the problem of finding a minimum set of $s$-clubs that covers the input graph.

The identification of Top-k-2-clubs turns to be NP-hard (as Max-2-clubs is NPhard), for this reason we design a genetic algorithm based heuristic by defining: first, a specific set of search operators for obtaining GA's approximate solutions, then a greedy approach to extrapolate the $\mathrm{k}$ top different approximations. While GA optimization is not new in literature, the interest on designing new heuristics and special operators for the applied models is still required to deal with the intractability of computational problems, with new applications in different contexts $[9,23]$.

The paper is organized as follows. In Section 2 we provide the definitions and we introduce the problem we are interested in. In Section 3, we discuss the GA-based approach to seek approximate solutions for the Top-k-2-clubs. In Section 4, we report numerical evaluations based on Erdös-Rényi random graphs. Section 5 discusses the preliminary results and the future development of our research.

ICCS Camera Ready Version 2019

To cite this paper please use the final published version:

DOI $10.1007 / 978-3-030-22750-0 \_63$ 


\section{Preliminaries}

Let $G=(V, E)$ be a graph, and $V^{\prime} \subseteq V$ a subset of the input vertices $V$. Denote by $G\left[V^{\prime}\right]$ the subgraph of $G$ induced by $V^{\prime}$, and $d_{G}(u, v)$ the distance (i.e., length of a shortest path) between two vertices $u, v$. The diameter of $G=(V, E)$ is defined as $\max _{u, v \in V} d_{G}(u, v)$, i.e., the maximum distance between any two vertices in $V$.

Given a graph $G=(V, E)$, a 2-club in $G$ is a subgraph $G[W]$, with $W \subseteq V$, that has diameter of at most 2 . The property of being a 2-club is not hereditary ${ }^{4}$. This means that if a graph $G$ is a 2-club, then a subgraph of $G$ is not necessarily a 2-club (see Fig. 1).

We introduce now the formal definition of the problem, denoted as Top-k-2-clubs. The Top-k-2-clubs maximizes an objective function that considers the size of the 2-clubs in the solution (since we want to compute large 2-clubs) and a distance function dist to provide graphs that are significantly different. The parameter $\lambda$ allows us to define how much relevance has the distance with respect to the size of the 2-clubs.

Problem 1 Top-k-2-club

Input: a graph $G=(V, E)$, a value $\lambda>0$.

Output: a set $\mathcal{W}=\left\{G\left[W_{1}\right], \ldots, G\left[W_{k}\right]\right\}$ of $k$ 2-clubs, with $1 \leq k<|V|$ and $W_{i} \subseteq V$, that maximizes the following value

$$
r(\mathcal{W})=\sum_{i=1}^{k}\left|W_{i}\right|+\lambda \sum_{i=1}^{k-1} \sum_{j=i+1}^{k} \operatorname{dist}\left(G\left[W_{i}\right], G\left[W_{j}\right]\right)
$$

where

$$
\operatorname{dist}\left(G\left[W_{i}\right], G\left[W_{j}\right]\right)= \begin{cases}2-\frac{\left|W_{i} \cap W_{j}\right|^{2}}{\left|W_{i}\right|\left|W_{j}\right|} & \text { if } W_{i} \neq W_{j}, \\ 0 & \text { else. }\end{cases}
$$

Notice that Top-k-2-clubs, when $k=1$, is exactly Max-2-club. Since Max-2-club on an input graph $G=(V, E)$ is NP-hard [4] and not approximable within factor $|V|^{1 / 2-\varepsilon}$, for each $\varepsilon>0$ [2], the same properties hold for Top-k-2-clubs.

\section{A Genetic Algorithm for the Top-k-s-club Problem}

As reported above, the Top-k-2-clubs is NP-hard, thus making optimization potentially impracticable. Our approach here is to provide approximate solutions by designing dedicated genetic operators. Let $G\left[V^{\prime}\right]$ be a 2-club of the input graph $G=(V, E)$, for some set of vertices $V^{\prime} \subseteq V$. We represent GA's solutions as binary chromosomes $c$, of size $|V|$, such that for each $v_{i} \in V^{\prime}, c[i]=1\left(c[i]=0\right.$ if $\left.v_{i} \in V \backslash V^{\prime}\right)$. With a slight abuse of notation, we denote by $G[c]$ the subgraph of $G$ induced by the representation of chromosome $c$. Furthermore, $V[c]$ and $E[c]$ represent the set of vertices, $V^{\prime}$, and edges of $G[c]=G\left[V^{\prime}\right]$. With the given representation, a set of $k$ chromosomes, which are involved in the offspring generation, is interpreted as a set of hypothesis of feasible solutions (i.e., hypotheses of potential 2-clubs). To quantify the validity of such (assertions) hypothesis, chromosomes will be then evaluated by the fitness function.

\footnotetext{
${ }^{4}$ This property can be extended to each $s \geq 2$.
} 


\subsection{Fitness Function}

We design the fitness function to promote new offspring in such a way that "feasible" chromosomes, able to properly express 2 -clubs (i.e., with a correct diameter value $\leq 2$ ) will gradually adapt through specific mutation and crossover operators.

Consider a chromosome $c$, and the graph $G=(V, E)$. In order to apply a fitness function which promotes the representation of large subgraphs $G[c]$, when $0 \leq \operatorname{diam} \leq$ 2 , we consider the following.

$$
f\left(n_{v}, \operatorname{diam} ; S\right)= \begin{cases}n_{v} & \text { if } 0 \leq \operatorname{diam} \leq 2 ; \\ \frac{1}{n_{v}} & \text { if } 2<\operatorname{diam}\end{cases}
$$

where $n_{v}$ is the number of vertices of $G[c]$.

Notice that, when diam $>2$ (i.e., unfeasible solutions) we obtain fitness values which decrease asymptotically for large subgraphs with size $n_{v}$, thus penalizing the corresponding chromosomes.

\subsection{Mutation}

The following types of mutations are considered with equal probability.

- Mutation 1. In this case, mutation is applied to correct hypotheses sparingly and consistently. For this, consider the set $V_{+}=\left\{v_{i}: c[i]=1\right\}$ and the associated graph $G\left[V_{+}\right]$, which should correspond to some feasible 2-club. In order to check such a feasibility, we randomly sample a vertex $v^{\prime} \in V_{+}$, and for each pair $\left(v_{i}, v^{\prime}\right), v_{i} \in$ $V_{+} \backslash\left\{v^{\prime}\right\}$ we verify whether the minimum length between $v_{i}$ and $v^{\prime}$ is of at most 2 . If this is not the case, $c[i]$ is flipped to 0 .

- Mutation 2. Similarly to the previous case this operator has the objective to sparingly increment the size of a solution. We consider now $V_{-}=\left\{v_{j}: c[j]=0\right\}$ and the current subgraph $G\left[V_{+}\right]$induced by $c$. In order to consistently add vertices to $V_{+}$, we sample some $v^{\prime}$ from $V_{-}$(equivalently, we will have some $j^{\prime}$ such that $\left.v^{\prime}=v_{j^{\prime}} \in V_{-}: c\left[j^{\prime}\right]=0\right)$ to check whether the shortest distance of $v^{\prime}$ from vertices in $V_{+}$is not larger than 2. In this case, the corresponding bit $c\left[j^{\prime}\right]$ is flipped to 1 .

- Standard Mutation. This is a standard mutation procedure where bits of the selected chromosome are randomly switched on or off.

\subsection{Cross-over}

The cross-over operations operations are selected with equal probability.

- Standard cross-over. Parts of parents' chromosomes are copied and mixed in new offspring with standard one-point crossover.

- Logical AND/OR cross-over. New offspring are generated by applying logical AND and logical OR operations between parents. 
Table 1. Performances: Models; Av. Fitness (Fit); Av. Inp. Diameter (InD); Av. Out. Diameter (OutD); Av. Jaccard (AvJ); Av. Covering (AvC); Av. User (T1) and System Time (T2)

\begin{tabular}{llllllll}
\hline Models & \multicolumn{1}{c}{ AvFit } & InD & OutD & AvJ & AvC & T1 & T2 \\
ER(100,0.1) & 12.4 & 4 & 2 & 0.31 & 0.13 & 98.4 & 1.30 \\
ER(150,0.1) & 16.8 & 3 & 2 & 0.47 & 0.12 & 154 & 1.86 \\
ER(200,0.1) & 27.1 & 3 & 2 & 0.69 & 0.14 & 233 & 2.49 \\
ER(300,0.1) & 35.1 & 3 & 2 & 0.74 & 0.12 & 370 & 3.02 \\
ER(400,0.1) & 41.1 & 3 & 2 & 0.78 & 0.11 & 453 & 3.90 \\
\hline \hline
\end{tabular}

\subsection{Selection of Top $k$ 2-clubs}

The final step of the algorithm selects $k$ 2-clubs (chromosomes) from the population. Denote by $\mathcal{W}$ the solution of Top- $k$-2-clubs we are computing. We apply a greedy procedure consisting of $k$ iterations. In the first iteration, a 2 -club of the population (denoted by $G\left[W_{1}\right]$ ) having maximum cardinality is added to $\mathcal{W}$.

Consider iteration $t$, with $2 \leq t \leq k$, of the greedy procedure. Assume that $\mathcal{W}=$ $\left\{G\left[W_{1}\right], \ldots, G\left[W_{t-1}\right]\right\}$, iteration $t$ adds to $\mathcal{W}$ a 2 -club $G\left[W_{t}\right]$ of the population that maximizes the following value

$$
\left|W_{t}\right|+\lambda \sum_{i=1}^{t-1} \sum_{j=i+1}^{t} \operatorname{dist}\left(G\left[W_{i}\right], G\left[W_{j}\right]\right)
$$

\section{Numerical Experiments}

The goal of our experiments was to check the capability of GAs to provide feasible solutions in reasonable computational time. The whole procedure described above was coded in R using the "GA" package [22]. In this work we use synthetic data by sampling Erdos-Renyi (ER) random graphs, $E R(n, p=0.1)$, with different number of vertices, $n$ [5].

To ensure both the correctness of the Genetic Algorithm and the computational tractability of our approach, we followed a standard practice of the evolutionary methods; that is, maintaining the tractability of genetic operators while promoting, at the same time, new evaluable offspring, which in our case provide feasible diameter values for the obtained solutions.

Similarly, the termination of the genetic algorithm is guaranteed by standard criteria. We have set both the revaluation number of the fitness with respect to new populations (equivalently, the number of GA iterations) and the number of new consecutive generation without fitness improvement. Finally, to get a more robust performance evaluation, each Erdos graph was repeatedly sampled 3 times. Performances are reported in Tab. 1. The following comments summarize our results.

1. All models are correct, being (2-clubs) with diameter $\leq 2$.

2. Since the considered problem is computationally intractable, it is not possible to compare the optimal solution with those provided by the described approach. In order to give, at least, a qualitative idea of the validity of the obtained approximations, 
we considered both the average covering (output / input vertices) of the input graph (obtained through the returned solutions, i.e., 10 largest solutions) and a measure of how such solutions differ from each other. In this way, the higher the number of covering ratio (and the more dissimilar are the solutions), the more input graph cover is qualitatively effective. To this aim, the Jaccard index was applied. Specifically, we remind that the smaller this index, the more dissimilar are the solutions. Observing table 1 more convincing solutions seem to be those obtained for a small number of input vertices. Moreover, the coverage performances do not decrease in an "obvious" way in the considered models.

3. Covering performances are reported. In this case, we get, on average, a covering value at least of $10 \%$.

4. A reasonable system time is observed after execution ( $\mathrm{T} 2 \leq 4$ seconds).

\section{Conclusion}

Identifying cohesive subgraphs within a network is a typical task with many applications in different important fields. In this paper, we reported our work in progress by considering the case where a collection of Top-k-2-clubs (i.e., largest different cohesive subgraphs) is maximized, providing large communities of a network covering. The computational hardness of the problem makes it impracticable to get optimal solutions. Here, we designed a set of dedicated GA operators to return approximate solutions at reasonable costs.

The preliminary results reported in this paper show we can get correct solutions in a reasonable time. Although compelling solutions seem to be provided for small graphs only ( $n \leq 150$ ), the $10 \%$ of the input graph size is covered.

Some aspects of this research can certainly be considered in a future extension. For example, a detailed analysis should be applied to optimize the parameters of the applied models. Although different igraph (R package) default values (i.e., probabilities of the search operators) have been used, accurate tuning should be properly considered to evaluate performance improvement. In this case, for example, the irace $\mathrm{R}$ package [17] can be easily applied for this purpose. Furthermore, only $s-c l u b s$ with $s=2$ were considered. Our approach can further be scaled up to any value $s \geq 2$ and $k$ (number of 2-clubs). Finally, real case analysis cannot be neglected in this research, and this will be another extension for a future version of the work.

Acknowledgments This work was partially supported by national funds through FCT (Fundação para a Ciência e a Tecnologia) under project DSAIPA/DS/0022/2018 (GAD$\mathrm{gET})$.

\section{References}

1. Alba, R.D.: A graph-theoretic definition of a sociometric clique. Journal of Mathematical Sociology 3, 113-126 (1973)

2. Asahiro, Y., Doi, Y., Miyano, E., Samizo, K., Shimizu, H.: Optimal Approximation Algorithms for Maximum Distance-Bounded Subgraph Problems. Algorithmica (2017)

ICCS Camera Ready Version 2019

To cite this paper please use the final published version:

\begin{tabular}{lll} 
DOI & $10.1007 / 978-3-030-22750-0 \_63$ \\
\hline
\end{tabular} 
3. Balasundaram, B., Butenko, S., Trukhanov, S.: Novel approaches for analyzing biological networks. J. Comb. Optim. 10(1), 23-39 (2005)

4. Balasundaram, B., Butenko, S., Trukhanov, S.: Novel approaches for analyzing biological networks. J. Comb. Optim. 10(1), 23-39 (2005)

5. Bollobas, B.: Random Graphs. Cambridge University Press (2001)

6. Bourjolly, J., Laporte, G., Pesant, G.: An exact algorithm for the maximum k-club problem in an undirected graph. European Journal of Operational Research 138(1), 21-28 (2002)

7. Chang, M., Hung, L., Lin, C., Su, P.: Finding large k-clubs in undirected graphs. Computing 95(9), 739-758 (2013)

8. Dondi, R., Mauri, G., Sikora, F., Zoppis, I.: Covering with clubs: Complexity and approximability. In: Iliopoulos, C.S., Leong, H.W., Sung, W. (eds.) Combinatorial Algorithms - 29th International Workshop, IWOCA 2018, Singapore, July 16-19, 2018, Proceedings. Lecture Notes in Computer Science, vol. 10979, pp. 153-164. Springer (2018)

9. Dondi, R., Mauri, G., Zoppis, I.: Orthology correction for gene tree reconstruction: Theoretical and experimental results. Procedia Computer Science 108, 1115-1124 (2017)

10. Dondi, R., Mauri, G., Zoppis, I.: On the tractability of finding disjoint clubs in a network. Theoretical Computer Science In press (2019). https://doi.org/10.1016/j.tcs.2019.03.045

11. Galbrun, E., Gionis, A., Tatti, N.: Top-k overlapping densest subgraphs. Data Min. Knowl. Discov. 30(5), 1134-1165 (2016). https://doi.org/10.1007/s10618-016-0464-z

12. Golovach, P.A., Heggernes, P., Kratsch, D., Rafiey, A.: Finding clubs in graph classes. Discrete Applied Mathematics 174, 57-65 (2014)

13. Hartung, S., Komusiewicz, C., Nichterlein, A.: Parameterized algorithmics and computational experiments for finding 2-clubs. J. Graph Algorithms Appl. 19(1), 155-190 (2015)

14. Komusiewicz, C.: Multivariate algorithmics for finding cohesive subnetworks. Algorithms 9(1), 21 (2016)

15. Komusiewicz, C., Sorge, M.: An algorithmic framework for fixed-cardinality optimization in sparse graphs applied to dense subgraph problems. Discrete Applied Mathematics 193, 145-161 (2015)

16. Laan, S., Marx, M., Mokken, R.J.: Close communities in social networks: boroughs and 2-clubs. Social Netw. Analys. Mining 6(1), 20:1-20:16 (2016)

17. Lpez-Ibez, M., Dubois-Lacoste, J., Prez Cceres, L., Sttzle, T., Birattari, M.: The irace package: Iterated racing for automatic algorithm configuration. Operations Research Perspectives 3, 43-58 (2016). https://doi.org/10.1016/j.orp.2016.09.002

18. Mokken, R.: Cliques, clubs and clans. Quality \& Quantity: International Journal of Methodology 13(2), 161-173 (1979)

19. Mokken, R.J., Heemskerk, E.M., Laan, S.: Close communication and 2-clubs in corporate networks: Europe 2010. Social Netw. Analys. Mining 6(1), 40:1-40:19 (2016)

20. Pasupuleti, S.: Detection of protein complexes in protein interaction networks using n-clubs. In: Evolut. Comput., Machine Learning and Data Mining in Bioinformatics, 6th EvoBIO 2008, Naples, Italy, March 26-28, 2008. Proceedings. pp. 153-164 (2008)

21. Schäfer, A., Komusiewicz, C., Moser, H., Niedermeier, R.: Parameterized computational complexity of finding small-diameter subgraphs. Optimization Letters 6(5), 883-891 (2012)

22. Scrucca, L.: GA: A package for genetic algorithms in R. Journal of Statistical Software 53(4), 1-37 (2013)

23. Weise, T., Zapf, M., Chiong, R., Nebro, A.J.: Why is optimization difficult? In: NatureInspired Algorithms for Optimisation, pp. 1-50. Springer (2009)

ICCS Camera Ready Version 2019

To cite this paper please use the final published version:

DOI $10.1007 / 978-3-030-22750-0 \_63$ 